\title{
Can More Aggressive Treatment Improve Prognosis in Patients with Hepatocellular Carcinoma? A Direct Comparison of the Hong Kong Liver Cancer and Barcelona Clinic Liver Cancer Algorithms
}

Young-Sun Lee ${ }^{1}$, Yeon Seok Seo ${ }^{1}$, Ji Hoon Kim ${ }^{1}$, Juneyoung $\mathrm{Lee}^{2}$, Hae Rim Kim ${ }^{1}$, Yang Jae Yoo ${ }^{1}$, Tae Suk Kim ${ }^{3}$, Seong Hee $\mathrm{Kang}^{1}$, Sang Jun Suh ${ }^{1}$, Moon Kyung Joo ${ }^{1}$, Young Kul Jung ${ }^{1}$, Beom Jae Lee ${ }^{1}$, Hyung Joon Yim ${ }^{1}$, Jong Eun Yeon ${ }^{1}$, Jae Seon $\mathrm{Kim}^{1}$, Jong-Jae Park ${ }^{1}$, Soon Ho Um ${ }^{1}$, Young-Tae Bak ${ }^{1}$, and Kwan Soo Byun ${ }^{1}$

Departments of ${ }^{1}$ Internal Medicine, ${ }^{2}$ Biostatistics, Korea University College of Medicine, Seoul, and ${ }^{3}$ Department of Internal Medicine, Kangwon National University School of Medicine, Chuncheon, Korea

Background/Aims: In addition to the globally endorsed Barcelona Clinic Liver Cancer (BCLC) staging system, other algorithms or staging systems have been developed, including the Hong Kong Liver Cancer (HKLC) staging system. This study aimed to validate the HKLC staging system relative to the BCLC staging system for predicting survival for hepatocellular carcinoma (HCC) patients in Korea. Methods: From 2004 to 2013, 2,571 patients newly diagnosed with HCC were consecutively enrolled at three Korea University medical centers. Results: Both staging systems differentiated survival well $(p<0.001)$. However, 1-year and 3-year survival were predicted better using the HKLC system than the BCLC system (area under the receiver operating characteristic curve: 0.869 vs 0.856 for 1 year, $p=0.002 ; 0.841$ vs 0.827 for 3 years, $p=0.010$ ). In hypothetical survival curves, the HKLC system exhibited better median overall survival than the BCLC system (33.1 months vs 19.2 months). In evaluations of prognosis according to either BCLC or HKLC treatment guidelines, risk of death was reduced in the group following only HKLC guidelines compared with the group following only BCLC guidelines (hazard ratio, 0.601; 95\% confidence interval, 0.443 to $0.816 ; p=0.001$ ). Conclusions: Although both staging systems predicted and discriminated HCC prognoses well, the HKLC system showed more encouraging survival benefits than the BCLC system. (Gut Liver 2018;12:94-101)

Key Words: Carcinoma, hepatocellular; Barcelona Clinic Liver Cancer; Hong Kong Liver Cancer; Neoplasm staging

\section{INTRODUCTION}

Hepatocellular carcinoma (HCC) is a major cause of morbidity worldwide, with 782,000 newly developed cases in $2012 .{ }^{1}$ In addition to its high incidence rate, HCC is the second most common cause of cancer-related death due to its high mortality rate. HCC is characterized by significant heterogeneity among patients in terms of liver reserve function; tumor status; and etiology, including alcohol, hepatitis B virus (HBV) infection, hepatitis C virus (HCV) infection, and nonalcoholic fatty liver disease (NAFLD). ${ }^{2}$

Cancer staging provides essential information about the prognosis of individual patients and helps clinicians to select standard treatment. Unlike other solid tumors, in which only the TNM staging system is generally used, ${ }^{3}$ many staging systems have been developed for HCC. Currently, 10 HCC staging systems have been developed based on liver function, $\alpha$-fetoprotein (AFP), performance status, and tumor staging. ${ }^{2,4}$ However, there is no universal consensus regarding which staging system is the most reliable to predict prognosis or to make proper recommendations for therapeutic modalities. This is due to the heterogeneity of HCC in terms of clinical characteristics, biological nature, and etiology. Among these variables, the etiology of HCC shows clear distinctions based on regional differences. ${ }^{5}$ Because HCV infection and NAFLD are the main causes of HCC in Western countries, most patients in these regions have accompanying liver cirrhosis. ${ }^{6,7}$ In contrast, HBV infection is the main cause of HCC in Asian and African patients, resulting in preserved liver function in a decent number of these individuals. ${ }^{8}$ Therefore,

\footnotetext{
Correspondence to: Ji Hoon Kim

Division of Gastroenterology and Hepatology, Department of Internal Medicine, Korea University Guro Hospital, Korea University College of Medicine, 148 Gurodong-ro, Guro-gu, Seoul 08308, Korea

Tel: +82-2-2626-3011, Fax: +82-2-2626-1038, E-mail: kjhhepar@naver.com

Received on January 20, 2017. Revised on March 23, 2017. Accepted on April 24, 2017. Published online September 7, 2017

pISSN 1976-2283 eISSN 2005-1212 https://doi.org/10.5009/gnl17040

Young-Sun Lee and Yeon Seok Seo contributed equally to this work as first authors.

(a) This is an Open Access article distributed under the terms of the Creative Commons Attribution Non-Commercial License (http://creativecommons.org/licenses/by-nc/4.0) which permits unrestricted non-commercial use, distribution, and reproduction in any medium, provided the original work is properly cited.
} 
more aggressive treatment strategies have been considered for patients with HCC in Asian countries. ${ }^{9-12}$

The Barcelona Clinic Liver Cancer (BCLC) staging system is the most popular staging system globally because it provides not only classification but also therapeutic recommendations. ${ }^{13}$ However, the BCLC staging system was developed using a population predominantly consisting of HCV-infected patients, and most patients had significant accompanying cirrhosis. Moreover, because stage B and C patients were heterogeneous, there is a possibility that inadequate treatments were provided to patients at these stages. ${ }^{4}$

In 2014, the new Hong Kong Liver Cancer (HKLC) staging system was developed based on a cohort of Asian HCC patients. ${ }^{4}$ In this cohort, the most common etiology was HBV infection, and liver function was preserved in a large proportion of patients, as in the majority of Asian countries. Because this staging algorithm recommends more aggressive treatment than the BCLC algorithm, the HKLC staging system investigators insisted that it could better predict survival outcomes than the BCLC staging system. Although three validation studies were reported in France, ${ }^{14}$ China, ${ }^{15}$ and Taiwan, ${ }^{16}$ the results were not consistent. In the current study, therefore, we validated this new staging system in prediction of survival and prognosis with recommended therapy as compared to the BCLC staging system in patients with HCC from a Korean multicenter cohort.

\section{MATERIALS AND METHODS}

\section{Patients and diagnosis}

In this study, a total of 2,571 newly diagnosed HCC patients were consecutively enrolled at three hospitals of the Korea University Medical Center (Anam, Guro, and Ansan hospitals) between January 2004 and December 2013. Pediatric patients, pretreated patients at other hospitals, and patients lost to followup without any treatment were excluded. The diagnosis of HCC was made according to the latest guidelines of the European Association for the Study of the Liver and American Association for the Study of Liver Diseases at the time of diagnosis based on histology and dynamic liver imaging including computed tomography scans and magnetic resonance imaging.

\section{Data collection}

All data were extracted from medical records retrospectively. Demographic characteristics, laboratory data, tumor characteristics, initial treatment modality, and survival outcomes were obtained through extensive review of medical records. End of follow-up date was determined by the patient's final visit to the hospital. Patient mortality status was assessed by both chart review and issuance of a death certificate. For patients lost to follow-up, we searched the Korean Cancer Center database, which contains information regarding mortality of cancer patients.

\section{Statistics}

Summary statistics are presented as mean or frequency (percentage), as appropriate. The baseline characteristics of patients by etiology (HBV infection, HCV infection, and alcoholic liver disease) were compared using Pearson chi-square test for categorical variables and one-way analysis of variance for continuous variables. Kaplan-Meier survival analysis was performed to estimate overall survival probability, and the log-rank test was used to compare the probability between the BCLC and HKLC staging systems. Cox proportional hazard regression model was used to compare a hazard of death for HKLC only following group compared to BCLC only following group.

The discriminant ability of the staging systems was evaluated using receiver operating characteristic curves. The prognostic capability of the staging systems was directly compared using the area under the receiver operating characteristic curve (AUROC). To compare hypothetical curves between the staging systems, a random sampling method was used, as described previously. ${ }^{4}$ For the hypothetical BCLC curve, the mortality and survival duration of patients who were not treated according to the BCLC staging system were randomly replaced by survival data from patients who had the same BCLC and HKLC stage and were receiving treatment according to the BCLC staging system. The HKLC curve was generated in the same manner.

All statistical analyses were performed using SPSS version 20 (IBM Corp., Armonk, NY, USA), except for the comparison of ROC between staging systems, for which MedCalc for Windows, version 14.0 (MedCalc Software, Ostend, Belgium) was used. All reported $\mathrm{p}$-values were two-tailed, and $\mathrm{p}<0.05$ was considered the minimum level of statistical significance. For full methods, please see the SUPPLEMENTARY METHODS.

\section{RESULTS}

\section{Baseline characteristics}

A total of 2,571 HCC patients were consecutively diagnosed with HCC at three university hospitals between 2004 and 2013. After the exclusion of pediatric patients, those pretreated at other hospitals, and those lost to follow-up without treatment, 2,211 patients were included in the analysis (Table 1).

The most common etiology of HCC was HBV infection $(1,523$, 68.9\%), followed by HCV infection (256, 11.6\%) and alcohol consumption (241, 10.9\%). According to etiologic factors, there were significant differences in demographic and tumor characteristics including age, sex, ECOG performance status, ChildPugh class, tumor size, number of tumors, tumor status, and vascular invasion (Table 1).

As initial treatment, 733 patients (33.2\%) received curative therapy including surgical resection $(296,13.4 \%)$, radiofrequency ablation (RFA) (425, 19.2\%), and liver transplantation (LT) (12, $0.5 \%$ ). Transarterial chemoembolization (TACE) was the most 
Table 1. Baseline Characteristics of the Study Population

\begin{tabular}{|c|c|c|c|c|c|}
\hline Characteristic & $\begin{array}{c}\text { All } \\
(\mathrm{n}=2,211)\end{array}$ & $\begin{array}{c}\text { HBV } \\
(\mathrm{n}=1,523,68.9 \%)\end{array}$ & $\begin{array}{c}\text { HCV } \\
(n=256,11.6 \%)\end{array}$ & $\begin{array}{c}\text { Alcohol } \\
(\mathrm{n}=241,10.9 \%)\end{array}$ & $\mathrm{p}$-value \\
\hline Age, yr & 58.3 & 54.9 & 67.0 & 62.3 & $<0.001$ \\
\hline Male sex & $1,757(79.5)$ & 1,232 (80.9) & $169(66.0)$ & 233 (96.7) & $<0.001$ \\
\hline ECOG PS & & & & & $<0.001$ \\
\hline \multirow[t]{2}{*}{$0 / 1$} & $1,691 / 241$ & $1,180 / 165$ & $210 / 23$ & $164 / 31$ & \\
\hline & (76.5/10.9) & (77.5/10.8) & (82.0/9.0) & $(68.1 / 12.9)$ & \\
\hline \multirow[t]{2}{*}{$2 / 3 / 4$} & $194 / 60 / 25$ & $129 / 37 / 12$ & $15 / 2 / 6$ & $30 / 14 / 2$ & \\
\hline & $(8.8 / 2.7 / 1.1)$ & $(8.5 / 2.4 / 0.8)$ & $(5.9 / 0.8 / 2.3)$ & $(12.4 / 5.8 / 0.8)$ & \\
\hline Child-Pugh & & & & & $<0.001$ \\
\hline \multirow[t]{2}{*}{$\mathrm{A} / \mathrm{B} / \mathrm{C}$} & $1,311 / 657 / 243$ & $949 / 412 / 162$ & $139 / 95 / 22$ & $114 / 87 / 40$ & \\
\hline & (59.3/29.7/11.0) & $(62.3 / 27.1 / 10.6)$ & $(54.3 / 37.1 / 8.6)$ & $(47.3 / 36.1 / 16.6)$ & \\
\hline Tumor size, cm & & & & & $<0.001$ \\
\hline \multirow[t]{2}{*}{$\leq 2 / 2-5 />5$} & $631 / 724 / 856$ & $428 / 471 / 624$ & $92 / 111 / 53$ & $73 / 83 / 85$ & \\
\hline & $(28.5 / 32.8 / 38.7)$ & $(28.1 / 30.9 / 41.0)$ & (35.9/43.4/20.7) & $(30.3 / 34.4 / 35.3)$ & \\
\hline No. of tumors & & & & & 0.031 \\
\hline \multirow[t]{2}{*}{$1 / 2-3 />3$} & $1,115 / 529 / 567$ & $776 / 351 / 396$ & $143 / 68 / 45$ & $112 / 62 / 67$ & \\
\hline & $(50.4 / 23.9 / 25.7)$ & (51.0/23.0/26.0) & $(55.9 / 26.6 / 17.5)$ & $(46.5 / 25.7 / 27.8)$ & \\
\hline Tumor status & & & & & $<0.001$ \\
\hline Early & $1,103(49.9)$ & $742(48.7)$ & $173(67.6)$ & $115(47.7)$ & \\
\hline Intermediate & $401(18.1)$ & $256(16.8)$ & 41 (16.0) & $53(22.0)$ & \\
\hline Locally advanced & 707 (32.0) & $525(34.5)$ & $42(16.4)$ & $73(30.3)$ & \\
\hline Vascular invasion & $599(27.1)$ & 385 (25.3) & $64(25.0)$ & $102(42.3)$ & $<0.001$ \\
\hline Metastasis & $199(9.0)$ & $142(9.3)$ & $15(5.9)$ & $23(9.5)$ & 0.155 \\
\hline Initial modality & & & & & $<0.001$ \\
\hline Resection & $296(13.4)$ & $226(14.8)$ & $21(8.2)$ & $24(10.0)$ & \\
\hline LT & $12(0.5)$ & $10(0.7)$ & 0 & $2(0.8)$ & \\
\hline RFA & $425(19.2)$ & $264(17.3)$ & $78(30.5)$ & 48 (19.9) & \\
\hline TACE & $1,018(46.0)$ & 715 (46.9) & $117(45.7)$ & $107(44.4)$ & \\
\hline Systemic therapy & $103(4.7)$ & $77(5.1)$ & $7(2.7)$ & $9(3.7)$ & \\
\hline Supportive therapy & $357(16.1)$ & $231(15.2)$ & 33 (12.9) & $51(21.2)$ & \\
\hline
\end{tabular}

Data are presented as number or number (\%).

HBV, hepatitis B virus; HCV, hepatitis C virus; ECOG PS, Eastern Cooperative Oncology Group performance status; LT, liver transplantation; RFA, radiofrequency ablation; TACE, transarterial chemoembolization.

common initial therapeutic modality, performed in 1,018 patients (46.0\%). A total of 103 patients (4.7\%) were treated with chemotherapy, and 357 patients (16.1\%) received supportive care.

\section{Survival outcomes according to BCLC and HKLC stages}

When patients were classified using the BCLC staging system, 289 patients (13\%) were categorized into stage 0; 722 patients (33\%) into stage A; 278 patients (12\%) into stage B; 635 patients (29\%) into stage C; and 287 patients (13\%) into stage D (Supplementary Fig. 1A). The BCLC staging system showed distinct overall survival outcomes for each of the stages $(p<0.001$ by log-rank test) (Fig. 1A). However, BCLC C and BCLC D showed similar survival in this population.

When the HKLC staging system was used, 735 patients (33\%) were included in stage I, 484 patients (22\%) in stage II, 290 patients (13\%) in stage III, 310 patients (14\%) in stage IV, and $392(18 \%)$ patients in stage V (Supplementary Fig. 1B). Overall survival results demonstrated that the HKLC staging system also showed distinct survival outcomes according to each stage ( $\mathrm{p}<0.001$ by log-rank test) (Fig. 1B).

\section{Prediction of survival outcomes between BCLC and HKLC staging systems}

Next, we checked the AUROC at 1 year and 3 years for both the BCLC and HKLC staging systems (Fig. 2). At 1 year, the AU- 
A

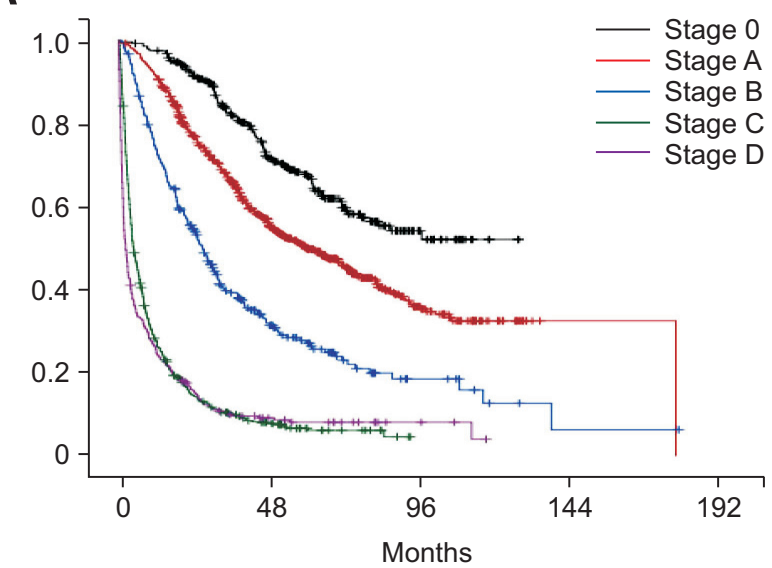

B

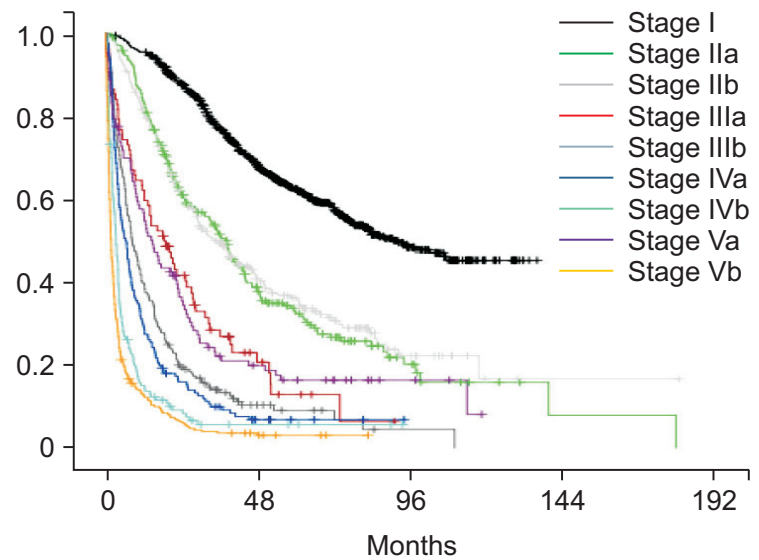

Fig. 1. Distinct survival outcomes for the Barcelona Clinic Liver Cancer (BCLC) and Hong Kong Liver Cancer (HKLC) staging systems. (A) KaplanMeier survival curve according to stages in the BCLC staging system (log-rank test, $p<0.001$ ). (B) Kaplan-Meier survival curve according to stages in the HKLC staging system (log-rank test, $\mathrm{p}<0.001)$.

A

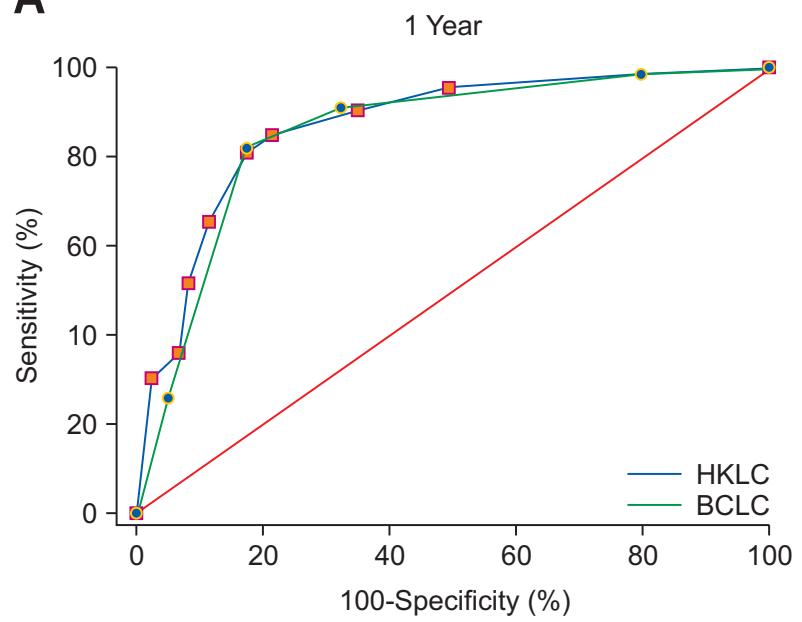

B

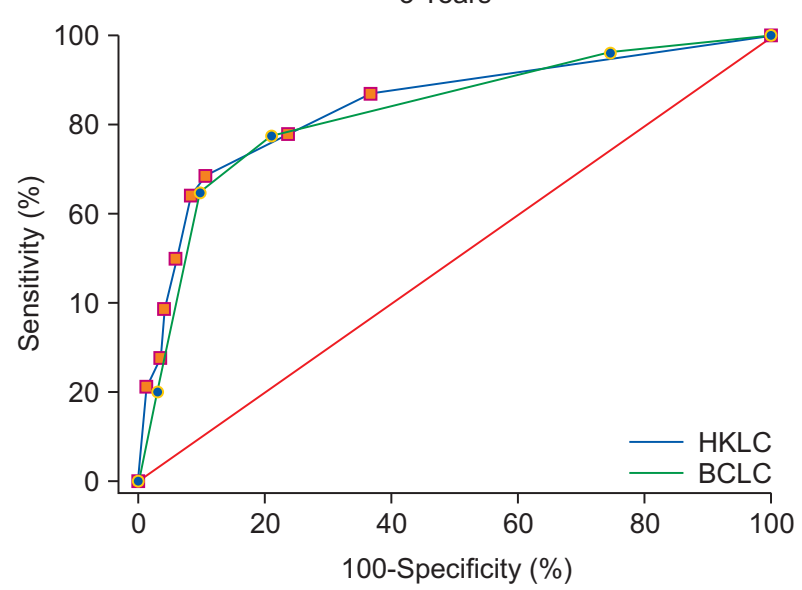

Fig. 2. Area under the receiver operating characteristic curves (AUROCs) at 1 year (A) and 3 years (B). The green line represents the AUROC for the Barcelona Clinic Liver Cancer (BCLC) staging system, whereas the blue line represents the AUROC for the Hong Kong Liver Cancer (HKLC) staging system.

ROC was 0.856 for BCLC (95\% confidence interval [CI], 0.840 to 0.870 ) and 0.869 for HKLC (95\% CI, 0.855 to 0.883). Although both staging systems showed good performance in predicting survival, the difference was statistically significant $(\mathrm{p}=0.002)$ (Supplementary Table 1). At 3 years, the AUROC was 0.827 for BCLC (95\% CI, 0.810 to 0.843) and 0.841 for HKLC (95\% CI, 0.825 to 0.857$)$. Although both staging systems showed good performance in predicting long-term survival, the difference was also statistically significant $(\mathrm{p}=0.010)$ (Supplementary Table 1$)$.

\section{Overall survival according to following BCLC and HKLC recommendations}

Through hypothetical Kaplan-Meier curves, we compared the overall survival between two groups that were treated according to the staging system used, BCLC or HKLC. Because these were hypothetical curves, it is impossible to perform statistical comparative analysis between the BCLC and HKLC staging systems. Nevertheless, the overall median survival was relatively higher in the HKLC staging system (33.1 months) than in the BCLC staging system (19.2 months) (Fig. 3).

\section{Cross table according to BCLC stage, HKLC stage, and initial treatment modality}

In Table 2, all patients are arranged by their BCLC stage, HKLC stage, and the treatment modality they received initially. Although some patients received initial therapy according to the recommendations of both the BCLC and HKLC staging systems, other patients were treated according to the recommendations of either the BCLC or HKLC staging system.

Among patients classified as stage B in the BCLC system and 


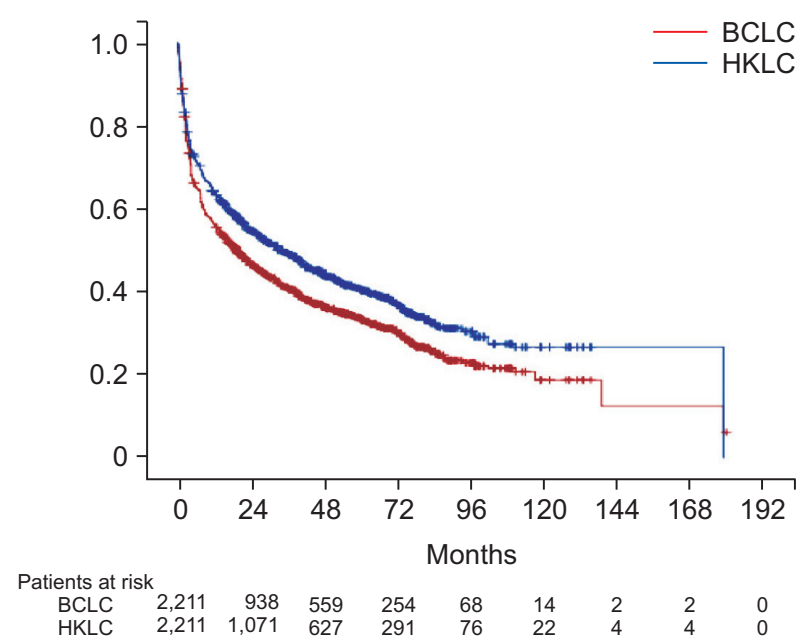

Fig. 3. Hypothetical Kaplan-Meier survival curves for the Barcelona Clinic Liver Cancer (BCLC, red line) and Hong Kong Liver Cancer (HKLC, blue line) staging systems. The overall median survival was 19.2 months for the BCLC staging system but 33.1 months for the HKLC staging system.
I/II in the HKLC system, some received TACE according to BCLC guidelines, while others received curative therapy according to HKLC recommendations. When overall survival was compared between these two groups, TACE-treated patients showed significantly decreased median overall survival compared to those who received curative treatment (25.9 months vs 69.3 months, $\mathrm{p}<0.001$ by log-rank test) (Supplementary Fig. 2A). Among patients classified as stage C in the BCLC system and II in the HKLC system, those who received systemic therapy according to the BCLC algorithm experienced significantly decreased median overall survival compared to those who were treated with curative therapy as indicated by the HKLC algorithm (7.5 months vs not available [NA], $\mathrm{p}<0.001$ by log-rank test) (Supplementary Fig. 2B). Likewise, in patients classified as stage $\mathrm{C}$ in the BCLC system and III in the HKLC system, systemic therapy according to BCLC guidelines led to decreased median overall survival compared to treatment with TACE as indicated by the HKLC algorithm (2.5 months vs 8.0 months, $\mathrm{p}=0.004$ by log-rank test) (Supplementary Fig. 2C).

Among patients classified as stage 0/A in the BCLC staging system and IIIa in the HKLC staging system, patients who received curative therapy according to the BCLC staging system

Table 2. Cross Table According to BCLC Stage, HKLC Stage, and Initial Therapeutic Modality

\begin{tabular}{|c|c|c|c|c|c|c|c|}
\hline BCLC stage & HKLC stage & Resection & Ablation & LT & TACE & Systemic therapy & Supportive care \\
\hline \multirow[t]{2}{*}{0} & I & $45^{*}$ & $94^{*}$ & $0^{*}$ & 93 & 0 & 0 \\
\hline & IIa & $5^{*}$ & $27^{*}$ & $0^{*}$ & 24 & 0 & 1 \\
\hline \multirow[t]{4}{*}{ A } & I & $132^{*}$ & $130^{*}$ & $2^{*}$ & 179 & 0 & 1 \\
\hline & IIa & $9^{*}$ & $66^{*}$ & $1^{*}$ & 82 & 0 & 2 \\
\hline & $\mathrm{IIb}$ & $39^{*}$ & $5^{\dagger}$ & $1^{\dagger}$ & 55 & 0 & 0 \\
\hline & IIIa & $3^{\dagger}$ & $2^{\dagger}$ & $0^{\dagger}$ & $13^{\ddagger}$ & 0 & 0 \\
\hline \multirow[t]{5}{*}{ B } & I & $17^{\ddagger}$ & $13^{\ddagger}$ & $1^{\ddagger}$ & $28^{\dagger}$ & 0 & 0 \\
\hline & IIa & $0^{\ddagger}$ & $6^{\ddagger}$ & $1^{\ddagger}$ & $6^{\dagger}$ & 0 & 0 \\
\hline & $\mathrm{IIb}$ & $16^{\ddagger}$ & 15 & 0 & $64^{\dagger}$ & 0 & 2 \\
\hline & IIIIa & 3 & 13 & 0 & $27^{*}$ & 0 & 2 \\
\hline & IIIlb & 4 & 3 & 0 & $53^{*}$ & 2 & 2 \\
\hline \multirow[t]{8}{*}{ C } & IIa & $0^{\ddagger}$ & $0^{\ddagger}$ & $2^{\ddagger}$ & 2 & $0^{\dagger}$ & 9 \\
\hline & $\mathrm{IIb}$ & $4^{\ddagger}$ & 8 & 0 & 25 & $2^{\dagger}$ & 5 \\
\hline & IIIa & 0 & 2 & 0 & $12^{\ddagger}$ & $0^{\dagger}$ & 5 \\
\hline & IIIb & 11 & 2 & 0 & $105^{\ddagger}$ & $3^{\dagger}$ & 23 \\
\hline & IVa & 2 & 7 & 0 & 84 & $44^{*}$ & 23 \\
\hline & $\mathrm{IVb}$ & 4 & 6 & 0 & 55 & $21^{*}$ & $64^{\ddagger}$ \\
\hline & $\mathrm{Va}$ & 0 & 2 & $0^{\ddagger}$ & 8 & $0^{\dagger}$ & 3 \\
\hline & $\mathrm{Vb}$ & 1 & 1 & 0 & 24 & $16^{\dagger}$ & $50^{\ddagger}$ \\
\hline \multirow[t]{2}{*}{$\mathrm{D}$} & $\mathrm{Va}$ & 1 & 18 & $4^{\ddagger}$ & 41 & 2 & $28^{\dagger}$ \\
\hline & $\mathrm{Vb}$ & 0 & 5 & 0 & 38 & 13 & $137^{*}$ \\
\hline
\end{tabular}

BCLC, Barcelona Clinic Liver Cancer; HKLC, Hong Kong Liver Cancer; LT, liver transplantation; TACE, transarterial chemoembolization.

${ }^{*}$ Number of patients who received treatment in accordance with both the BCLC and HKLC recommendations; ${ }^{\dagger}$ Number of patients who received treatment in accordance with only the BCLC recommendation; ${ }^{\ddagger}$ Number of patients who received treatment in accordance with only the HKLC recommendation. 
experienced increased median overall survival compared to those who were treated with TACE as indicated by the HKLC staging system (NA vs 13.9 months, $\mathrm{p}=0.003$ by log-rank test) (Supplementary Fig. 3).

\section{Survival outcomes according to following recommenda- tions}

We classified patients into three groups: one group following both the BCLC and HKLC algorithms; one group following only the BCLC algorithm; and one group following only the HKLC algorithm. Among them, the group following the recommendations of both staging systems showed significantly increased overall median survival (47.1 months) compared to the group following only BCLC recommendations (18.3 months, $\mathrm{p}<0.001$ by log-rank test) or only HKLC recommendations (6.3 months, $\mathrm{p}<0.001$ by log-rank test) (Supplementary Fig. 4). Between the group following only the BCLC algorithm and the group following only the HKLC algorithm, the BCLC only group demonstrated increased overall median survival significantly $(\mathrm{p}<0.001$ by log-rank test). However, the distribution of specific stages showed differences between the two groups; the group following only the BCLC recommendations had early stage patients, while the group following only the HKLC recommendations was dominated by late stage patients (Supplementary Table 2). After adjustment of the BCLC and HKLC stages, interestingly, the group following the HKLC algorithm only experienced reduced risk of death compared with the group following the BCLC algorithm only (hazard ratio [HR], 0.601; 95\% CI, 0.443 to 0.816 ; $\mathrm{p}=0.001$ ) (Supplementary Table 2).

Next, we checked survival outcomes according to following recommendations with adjustment of BCLC and HKLC stages in the major three etiologies of HCC, alcohol, HBV, and HCV. In patients with HCC resulting from alcohol consumption, the group following BCLC recommendations only and the group following HKLC recommendations only showed similar survival (HR, 0.983; 95\% CI, 0.372 to 2.600; p=0.972) (Supplementary Table 3). Among HCC patients with HBV, however, the group following the HKLC algorithm only showed significantly decreased risk compared to the group following the BCLC algorithm only (HR, 0.596; 95\% CI, 0.420 to 0.845; p=0.004) (Supplementary Table 3). In patients with HCC resulting from $\mathrm{HCV}$, the group following only HKLC recommendations showed a relatively better survival curve than the group following only BCLC recommendations (HR, 0.536; 95\% CI, 0.188 to 1.531 ; $\mathrm{p}=0.244$ ) (Supplementary Table 3).

\section{DISCUSSION}

Since the BCLC staging system was developed and has come into worldwide use, more aggressive treatment strategies have been urgently demanded because of recent advances in treatment modalities for HCC. The HKLC staging system was devel- oped using a large HCC cohort at a single center in Hong Kong. ${ }^{4}$ This staging system showed effectiveness for selection of more aggressive treatment in patients with HCC, superiority in prediction of survival, and increased ability to predict overall survival outcomes. Although several validation studies comparing the HKLC and BCLC staging systems have been performed, ${ }^{14-16}$ the results were inconsistent. In this validation study, we found some benefit of the HKLC staging system in ability to predict survival and prognosis, particularly in patients with HBV-associated HCC.

Two validation cohort studies that were based on Asian patients with HCC reported better performance of the HKLC staging system in predicting prognosis of patients with HCC, comparing to the BCLC staging system. A Chinese study included 668 patients from a single center, ${ }^{15}$ and it was composed almost entirely of HBV-associated HCC patients (97.5\%), similarly to the original group that developed the HKLC staging system (80.0\%). In a study in Taiwan, 3,182 patients from a single center were analyzed, and HBV (41\%) was not a unilaterally dominant etiologic factor for HCC compared to HCV (23\%). ${ }^{16}$ In particular, the Taiwan study conducted subgroup analysis after classifying patients based on the etiology of HCC; the HKLC staging system showed a significant benefit of therapeutic efficacy only in the group of HBV patients, although that result was from a hypothetical curve. Likewise, our results demonstrated that HCC patients with HBV showed a significant survival benefit when they followed the HKLC recommendations compared to the BCLC recommendations. According to a previous study, HBVassociated HCC patients showed better survival after resection or LT compared to HCV-associated HCC patients. ${ }^{17}$ Therefore, HBV predominant populations showed better survival outcomes when they followed the HKLC recommendations compared to when they complied with the BCLC recommendations.

On the other hand, a European HCC cohort study revealed that the HKLC staging system did not show better predictive ability for survival compared to the BCLC staging system. ${ }^{14}$ Likewise, in a subgroup analysis of HCV-associated HCC patients from the Taiwan study, hypothetical Kaplan-Meier curves did not show a difference in overall survival between the groups following BCLC and HKLC recommendations. ${ }^{16}$ Generally, patients with HCV-associated HCC have accompanying cirrhosis, so curative treatment is often impossible due to poor liver function. ${ }^{18}$ Although antiviral therapy against HCV has also been found to reduce the recurrence of HCC following surgery in a meta-analysis, ${ }^{19}$ low sustained virologic response rate, side effects, and contraindication in advanced cirrhosis were concerns. However, because of the recent use of direct-acting antivirals, even in patients with decompensated cirrhosis, ${ }^{20}$ deterioration of liver function and recurrence of HCC might be more reduced after curative therapy, and following HKLC recommendations would lead to greater survival benefits.

Due to its heterogeneity, modifications of BCLC that sub- 
classify the intermediate stage (BCLC B) have been proposed. ${ }^{21}$ Similarly, our data and the original HKLC group data showed heterogeneity in BCLC stages B and C. ${ }^{4}$ This heterogeneity led to discordance in treatment recommendations between the BCLC and HKLC staging systems in some patients. In terms of prognosis, overall survival was better when patients were treated according to recommendations that were congruous between the BCLC and HKLC staging systems compared to when patients were treated following only the recommendations of either the BCLC or HKLC system. Notably, the group following only the recommendations of the HKLC system experienced a reduced HR compared to the group following only BCLC recommendations after adjustment of staging status.

When the therapeutic recommendation differed, the group following the HKLC recommendations showed better prognosis compared to the group following the BCLC recommendations in most cases. Patients with BCLC B and HKLC I/II had multinodular tumors; the largest was greater than $3 \mathrm{~cm}$ and the number was more than 3. The HKLC following group underwent more aggressive resection, resection with intraoperative RFA, or RFA targeting of tumors larger than $3 \mathrm{~cm}$. Patients with BCLC C and HKLC II had Eastern Cooperative Oncology Group performance status (ECOG PS) 1 or branched portal vein thrombosis. Among these patients, the HKLC following group underwent aggressive resection or RFA. Patients with BCLC C and HKLC III had portal vein thrombosis. Among these patients, the HKLC following group received TACE despite portal vein thrombosis. In all of these cases, the HKLC following group received more aggressive treatment than the BCLC following group, resulting in better prognosis. In the cases of BCLC A and HKLC IIIIa, however, the group following the BCLC algorithm that underwent surgical resection showed better survival compared to the group following the HKLC algorithm that received TACE. The patients in BCLC A and HKLC IIIa had a single large HCC (larger than $5 \mathrm{~cm}$ ) with Child-Pugh class B and ECOG PS 0. Although there have been some arguments regarding whether a single large HCC should be classified into BCLC A or $\mathrm{B}^{22,23}$ and the Taiwan group defined a single large $\mathrm{HCC}$ as BCLC $\mathrm{B},{ }^{16}$ we found that patients with single large HCCs showed better prognosis when they received surgical resection.

There are some weaknesses in the HKLC algorithm. First, the HKLC staging system is very complex compared to the BCLC staging system; the HKLC system consists of nine stages including substages, whereas the BCLC staging system consists of five stages including stage 0 . In addition, since the HKLC staging system was based on a population predominantly composed of HBV patients, there was limitation for application to other populations.

A major limitation of this validation study is that it was a retrospective study. Because the therapeutic modality would be determined with consideration of a patient's individual situation, bias would be inevitable without randomization. Moreover, there was a relatively large number of patients that received TACE as initial treatment compared to Western countries, but this finding is similar in Korea and China. ${ }^{24}$ The survival of patients with BCLC C and BCLC D was not significantly different in our study, probably because more patients received LT for BCLC D than for BCLC C (1.4\% vs and 0.3\%). In addition, there is concern that the ECOG status would be underestimated in patients with BCLC C. Lastly, there was a major difference in terms of the scope of systemic therapy. The BCLC algorithm suggests use of only sorafenib in patients with advanced HCC, whereas the HKLC algorithm recommend systemic therapy including sorafenib and cytotoxic chemotherapy in patients with HKLC stage IVa and IVb. If patients who received cytotoxic chemotherapy were regarded as not having followed recommendations for BCLC C, the survival benefit of the HKLC staging system would be weaker. However, this adjustment is unfavorable to the HKLC staging system, which includes cytotoxic chemotherapy as systemic therapy. In our population, however, the survival of patients treated with sorafenib (2.6 months) was similar to that of patients treated with cytotoxic chemotherapy (3.9 months, $\mathrm{p}=0.093$ ).

In conclusion, although both the BCLC and HKLC staging systems well predict and discriminate the prognosis of HCC, the HKLC staging system showed more encouraging survival benefits than the BCLC staging system, especially in HBV-associated HCC. The HKLC staging system generally recommended more aggressive treatment modalities than the BCLC system. Moreover, in cases of following BCLC recommendations that were more aggressive than HKLC recommendations, the survival of patients following BCLC recommendations was superior to that of patients following HKLC recommendations. Therefore, the two systems are complementary, not alternative, and more individualized and aggressive therapy might lead to better outcomes in the treatment of patients with HCC.

\section{CONFLICTS OF INTEREST}

No potential conflict of interest relevant to this article was reported.

\section{ACKNOWLEDGEMENTS}

This research was supported by Basic Science Research Program through the National Research Foundation of Korea (NRF) (2015R1C1A1A01052360).

\section{REFERENCES}

1. Ferlay J, Soerjomataram I, Dikshit R, et al. Cancer incidence and mortality worldwide: sources, methods and major patterns in GLOBOCAN 2012. Int J Cancer 2015;136:E359-E386.

2. Meier V, Ramadori G. Clinical staging of hepatocellular carci- 
noma. Dig Dis 2009;27:131-141.

3. Edge SB, Compton CC. The American Joint Committee on Cancer: the 7th edition of the AJCC cancer staging manual and the future of TNM. Ann Surg Oncol 2010;17:1471-1474.

4. Yau T, Tang VY, Yao TJ, Fan ST, Lo CM, Poon RT. Development of Hong Kong Liver Cancer staging system with treatment stratification for patients with hepatocellular carcinoma. Gastroenterology 2014;146:1691-1700.e3.

5. Sanyal AJ, Yoon SK, Lencioni R. The etiology of hepatocellular carcinoma and consequences for treatment. Oncologist 2010;15 Suppl 4:14-22.

6. Fattovich G, Stroffolini T, Zagni I, Donato F. Hepatocellular carcinoma in cirrhosis: incidence and risk factors. Gastroenterology 2004;127(5 Suppl 1):S35-S50.

7. El-Serag HB, Rudolph KL. Hepatocellular carcinoma: epidemiology and molecular carcinogenesis. Gastroenterology 2007;132:25572576.

8. Lin CL, Kao JH. Risk stratification for hepatitis B virus related hepatocellular carcinoma. J Gastroenterol Hepatol 2013;28:10-17.

9. Chan SL, Mo FK, Johnson PJ, et al. Prospective validation of the Chinese University Prognostic Index and comparison with other staging systems for hepatocellular carcinoma in an Asian population. J Gastroenterol Hepatol 2011;26:340-347.

10. Hsu CY, Liu PH, Lee YH, et al. Aggressive therapeutic strategies improve the survival of hepatocellular carcinoma patients with performance status 1 or 2: a propensity score analysis. Ann Surg Oncol 2015;22:1324-1331.

11. Kim JY, Sinn DH, Gwak GY, et al. Transarterial chemoembolization versus resection for intermediate-stage (BCLC B) hepatocellular carcinoma. Clin Mol Hepatol 2016;22:250-258.

12. Lee JM, Jang BK, Lee YJ, et al. Survival outcomes of hepatic resection compared with transarterial chemoembolization or sorafenib for hepatocellular carcinoma with portal vein tumor thrombosis. Clin Mol Hepatol 2016;22:160-167.

13. Llovet JM, Brú C, Bruix J. Prognosis of hepatocellular carcinoma: the BCLC staging classification. Semin Liver Dis 1999;19:329-338.
14. Adhoute X, Penaranda G, Bronowicki JP, Raoul JL. Usefulness of the HKLC vs. the BCLC staging system in a European HCC cohort. J Hepatol 2015;62:492-493.

15. Yan X, Fu X, Cai C, Zi X, Yao H, Qiu Y. Validation of models in patients with hepatocellular carcinoma: comparison of Hong Kong Liver Cancer with Barcelona Clinic Liver Cancer staging system in a Chinese cohort. Eur J Gastroenterol Hepatol 2015;27:1180-1186.

16. Liu PH, Hsu CY, Lee YH, et al. Hong Kong Liver Cancer staging system is associated with better performance for hepatocellular carcinoma: special emphasis on viral etiology. Medicine (Baltimore) 2015;94:e1772.

17. Franssen B, Alshebeeb K, Tabrizian P, et al. Differences in surgical outcomes between hepatitis B- and hepatitis C-related hepatocellular carcinoma: a retrospective analysis of a single North American center. Ann Surg 2014;260:650-656.

18. Hiotis SP, Rahbari NN, Villanueva GA, et al. Hepatitis B vs. hepatitis $\mathrm{C}$ infection on viral hepatitis-associated hepatocellular carcinoma. BMC Gastroenterol 2012;12:64.

19. Miao RY, Zhao HT, Yang HY, et al. Postoperative adjuvant antiviral therapy for hepatitis $\mathrm{B} / \mathrm{C}$ virus-related hepatocellular carcinoma: a meta-analysis. World J Gastroenterol 2010;16:2931-2942.

20. Ferenci P, Kozbial K, Mandorfer M, Hofer H. HCV targeting of patients with cirrhosis. J Hepatol 2015;63:1015-1022.

21. Bolondi L, Burroughs A, Dufour JF, et al. Heterogeneity of patients with intermediate (BCLC B) hepatocellular carcinoma: proposal for a subclassification to facilitate treatment decisions. Semin Liver Dis 2012;32:348-359.

22. Gao Q, Wang XY, Zhou J, Fan J. Heterogeneity of intermediatestage HCC necessitates personalized management including surgery. Nat Rev Clin Oncol 2015;12:10.

23. Forner A, Gilabert M, Bruix J, Raoul JL. Reply: heterogeneity of intermediate-stage HCC necessitates personalized management including surgery. Nat Rev Clin Oncol 2015;12:10.

24. Park JW, Chen M, Colombo M, et al. Global patterns of hepatocellular carcinoma management from diagnosis to death: the BRIDGE Study. Liver Int 2015;35:2155-2166. 